\title{
Facile synthesis of monodisperse thermally immiscible Ag-Ni alloy nanoparticles at room temperature
}

\author{
S TABATABAEI and S K SADRNEZHAAD* \\ Department of Materials Science and Engineering, Sharif University of Technology, P.O. Box 11365-9466, \\ Tehran, Iran
}

MS received 29 May 2013; revised 17 July 2013

\begin{abstract}
Ag and Ni are immiscible, mainly due to their large lattice mismatch. This paper reports on their nanoscale formation of solid solution at room temperature by simple reduction reactions which lead to the amorphous Ag-Ni alloy nanoparticles (ANPs) with mono-disperse distribution. Microscopic and spectroscopic studies confirmed dependence of the alloy composition on size of nanoparticles. In the presence of different ligands such as sodium citrate, polyvinyl alcohol and potassium carbonate a mixture of silver oxide and Ag-Ni ANPs was achieved. Stoichiometry of the Ag-Ni ANPs was also found to be strongly dependent on ligands of the reduction reaction and further study shows without any ligand $100 \%$ Ag-Ni ANPs was observed in the system. Using Tetrakis hydroxymethyl phosphonium chloride resulted in construction of near-uniform ANPs in the easily controllable conditions of the present alloying procedure. Nanoparticles having up to $65 \% \mathrm{Ni}$ were observed for the first time in this research.
\end{abstract}

Keywords. Chemical reduction; Ag-Ni alloying; nanoparticle; amorphous alloy.

\section{Introduction}

Nanocomposite Ag-Ni thin films have been used in protective coatings of electric connector terminals (Cheng and Hills 1997). Elemental Ag and Ni have long been known for their antibacterial properties. Complexes of $\mathrm{Ni}(\mathrm{II})$ have shown a distinct antibacterial behaviour against Staphylococcus aureus (Mondelli et al 2008) and Escherichia coli (Ghosh et al 1987) similar to Ag compounds (Sondi and Salopek-Sondi 2004; Morones et al 2005), which additionally display antibacterial properties towards Pseudomonas aeruginosa and Streptococcus pneumonia (Pattabi et al 2010). Ag-Ni ANPs are expected to demonstrate antibacterial effects against these germs due to their synergistic effects. Using Ag-Ni ANPs, at the same time, is more economical than pure silver ANPs. According to the literature, metal-catalyst decoration can substantially enhance hydrogen storage capacity of carbon substances (Kiran et al 2006). Storage capability of the Ag-Ni ANPs for hydrogen is inferable from reports on hydrogen adsorption of Ag-Rh nanoparticles (Kusada et al 2010).

Physical methods using high-energy sources like laser have been previously utilized to overcome energy barriers against non-equilibrium Ag-Ni ANP formation. For example, pulsed-laser irradiation is employed for vapour quenching of the $\mathrm{Ag}_{x} \mathrm{Ni}_{1-x}$ solid solution. The Ag content

*Author for correspondence (sadrnezh@sharif.edu) of the product has, however, not exceeded 44\% with this approach (Van Ingen et al 1994a). Using laser ablation for production of Ag-Ni thin film has also been reported (Van Ingen et al 1994b). No scalable/economical synthesis method has been conveyed for production of this material at room temperature. Physicochemical methods combining the application of high-energy radiation with chemical reaction has also been utilized for production of Ag-Ni ANPs before (Zhang et al 2010). More recently, a chemical procedure has been developed for the synthesis of Ag-Ni ANP via metal nitrates dissolution in ethylene glycol and subsequent reduction by hydrazine in the presence of polyethyleneimine (PEI) at $60{ }^{\circ} \mathrm{C}$ (Lee et al 2009).

To overcome positive enthalpy effects causing highly non-equilibrium alloying of immiscible elements at room temperature, excessive external energy is required. This energy, to some extent, may be provided by amorphous nanoscale $(\sim 4 \mathrm{~nm})$ alloying process. Details of the process - though not fully understood - are described as being related to the kinetic effects (Ma 2005). Immiscible systems of large positive enthalpies, such as Ag-Fe (Andrews and O’Brien 1992; Chinmay et al 2002), Ag-Ni (Kumar et al 2001; Gaudry et al 2003; Poondi and Singh 2000; and Zhang et al 2009), Cu-W (Gheorghe and Pantea 1997) and Cu-Ta (Kwon and Lee 1999), can be altered by high-energy treatments such as gamma radiation, laser ablation or heating methods. Ag and Ni have limited solubility even above $1445^{\circ} \mathrm{C}$. Their solution is, thus, unstable in bulk-scale level. According to the literature, only core-shell structure is thermodynamically stable at 
the nanoscale level (Liu et al 2008; Singelton and Nash 1987).

The current paper reports on synthesis of Ag-Ni ANPs by chemical reduction at room temperature by a simple chemical method. Compared with previously reported methods, the procedure introduced here is safer, scalable and more economical. Effect of the ligands on alloying process is also mentioned.

\section{Experimental}

Silver nitrate $\left(\mathrm{AgNO}_{3}\right)$, nickel nitrate $\left(\mathrm{NiN}_{2} \mathrm{O}_{6} \cdot 6 \mathrm{H}_{2} \mathrm{O}\right)$, sodium citrate $\left(\mathrm{Na}_{3} \mathrm{C}_{6} \mathrm{H}_{5} \mathrm{O}_{7}\right)$, polyvinyl alcohol (PVA) and potassium carbonate $\left(\mathrm{K}_{2} \mathrm{CO}_{3}\right)$ obtained from SigmaAldrich were used without further purification in this research. A solution containing $10^{-4} \mathrm{M}$ silver nitrate in $100 \mathrm{~mL}$ deionized water was prepared. Nickel nitrate $\left(10^{-4} \mathrm{M}\right)$ was added to the solution while stirring for 2 min, during which its colour remained unchanged. $\mathrm{NaBH}_{4}(0.01 \mathrm{~g})$ was added to the solution and stirred until grey colour appeared, indicating the formation of Ag-Ni alloy nanoparticles (ANPs). The experimental procedure is schematically depicted in figure 1 .

Selected area diffraction (SAD), energy dispersive X-ray spectroscopy (EDS), Gatan image filtering (GIF) and high angle annular dark field (HAADF) imaging were used to determine phase and structure of the Ag-Ni ANPs produced.

\section{Results and discussion}

Distributions of Ag and Ni in ANP are illustrated in figure 2(a and b). Based on atom probe tomography, Banerjee and Puthucode (2007) have reported spinodal structure

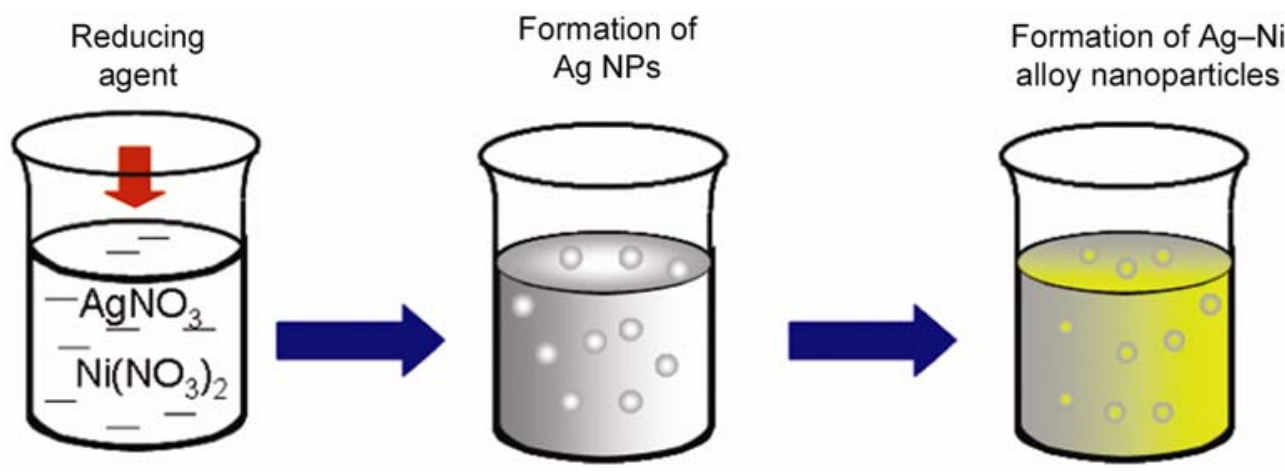

Figure 1. Co-reduction method for synthesis of Ag-Ni ANPs.
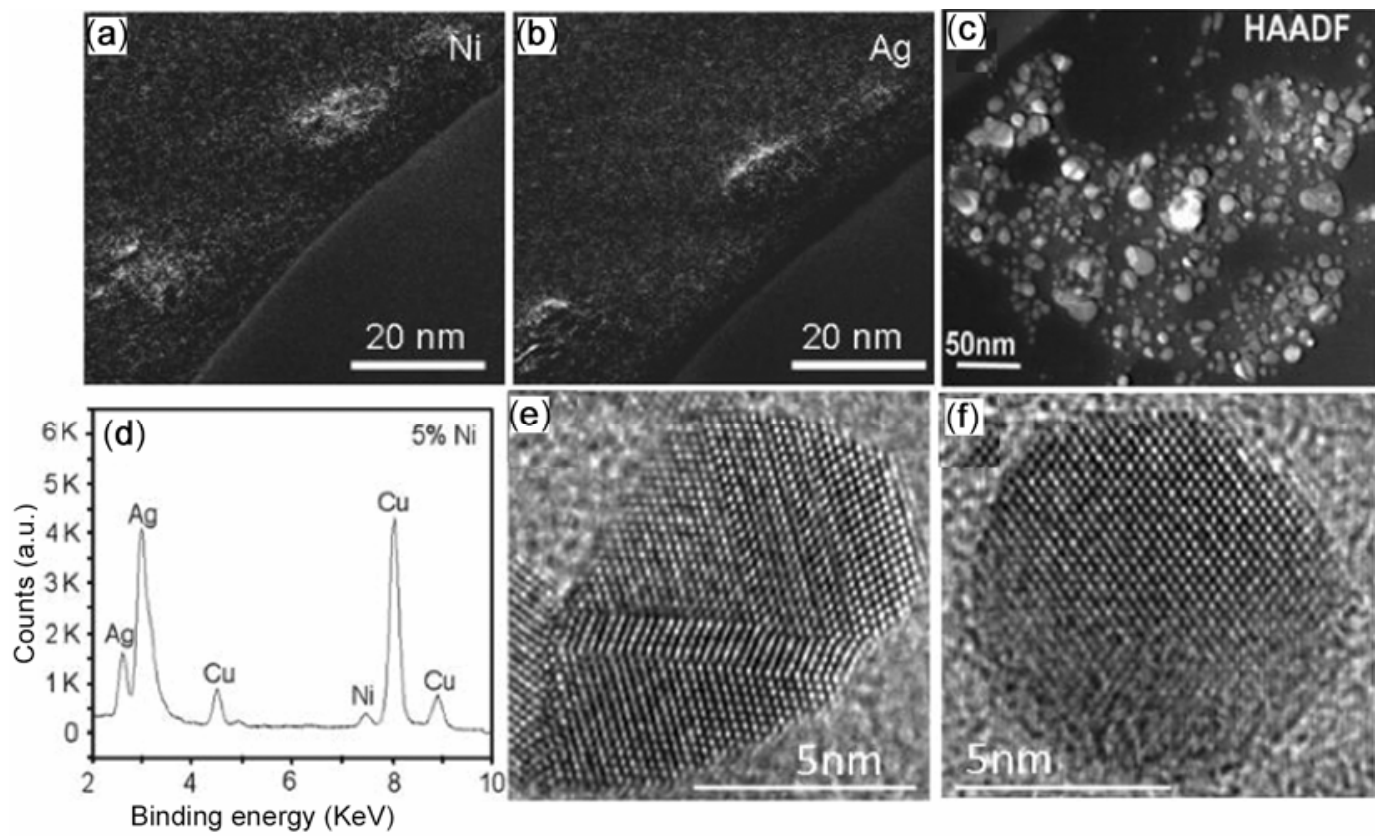

Figure 2. Gif confirms the existence of both $\mathrm{Ag}$ (a) and $\mathrm{Ni}(\mathbf{b})$ in an individual particle; HAAD image (c) shows that nanoparticles do not have a core-shell structure; EDS (d) reveals the existence of $5 \% \mathrm{Ni}$ in these particles and HRTEM images (e and $\mathbf{f}$ ) compare an Ag-Ni ANP with an Ag NP. 


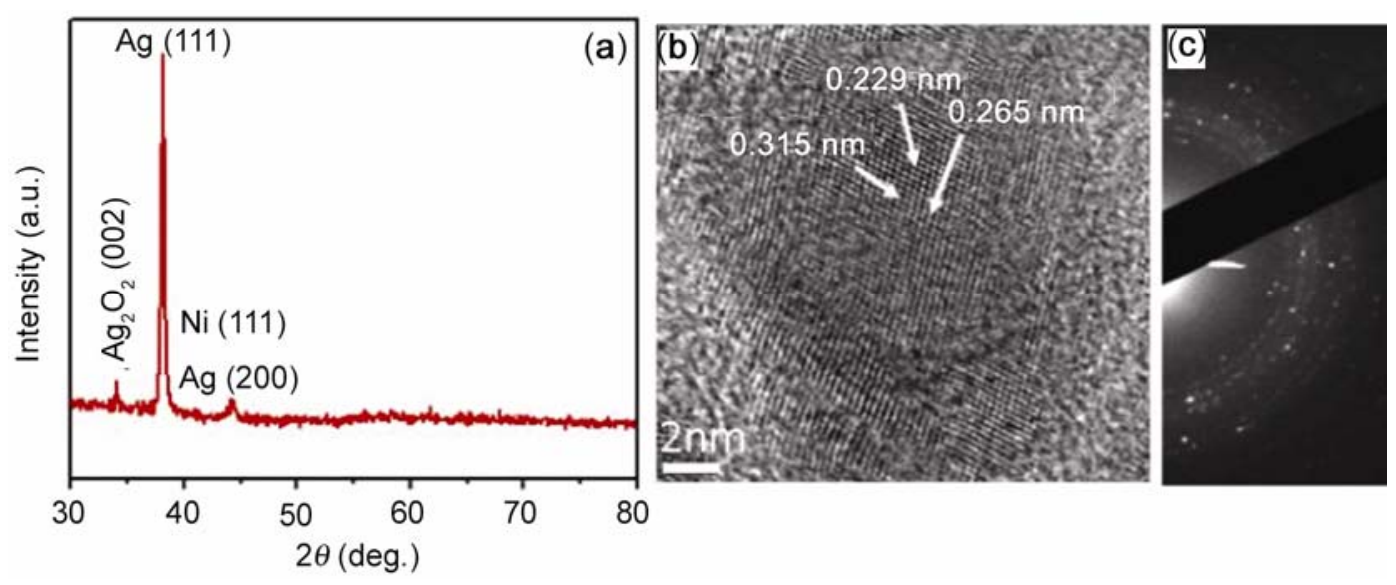

Figure 3. (a) XRD diagram, (b) HRTEM image and (c) SAD pattern of an Ag-Ni ANP in the presence of sodium citrate. Diffraction pattern confirms the presence of $\left(\begin{array}{lll}0 & 0 & 2\end{array}\right),\left(\begin{array}{lll}0 & 2 & 2\end{array}\right)$ and $\left(\begin{array}{ll}2 & 2\end{array}\right)$ planes of the silver oxide $\left(\mathrm{Ag}_{2} \mathrm{O}_{2}\right)$ and $\left(\begin{array}{lll}1 & 1 & 1\end{array}\right)$ and $\left(\begin{array}{lll}2 & 0 & 0\end{array}\right)$ planes of the Ag-Ni ANP.

rather than solid-solution configuration in the system. HAADF image of figure 2(c) shows no core-shell structure in the Ag-Ni ANPs produced in this research and, interestingly, some twins and multiple twins are observed in the system. EDS results confirm the existence of both $\mathrm{Ag}$ and $\mathrm{Ni}$ elements in an individual particle (figure 2d). High-resolution TEM (HRTEM) images of an Ag-Ni ANP and an Ag nanoparticle (NP) are provided in figure 2(e and f). Microscopic and spectroscopic data reconfirm dependence of the alloying process on the nanoscale particle sizes (Yasuda and Mori 1994; Shibata and Bunker 2002).

The XRD pattern shown in figure 3(a) demonstrates the characteristic peaks of an $\mathrm{Ag}-\mathrm{Ni}$ alloy nanoparticle. Small amounts of silver oxide $\left(\mathrm{Ag}_{2} \mathrm{O}_{2}\right)$ are observed in presence of the ligands. Figure 3(b) shows lattice fringes corresponding to an Ag-Ni ANP. Figure 3(c) displays the diffraction pattern of an $\mathrm{Ag}-\mathrm{Ni}$ ANP in the presence of sodium citrate. It shows (0 0 2), (0 $\left.\begin{array}{lll}0 & 2\end{array}\right)$ and (2 2 2) planes of the $\mathrm{Ag}_{2} \mathrm{O}_{2}$ and ( $\left(\begin{array}{lll}1 & 1 & 1\end{array}\right)$ and ( $\left(\begin{array}{lll}2 & 0 & 0\end{array}\right)$ planes of the Ag-Ni ANP present in the particle.

Further studies indicate a nearly $100 \%$ Ag-Ni ANP when no ligand is present; while stability remains a challenge. This indicates that ligands have a role to play in the ANP stability, and would intrude in the progress of the alloying process. Supporting information will be used in further discussion of this matter. SAD results from different parts of the samples reveal the presence of an amorphous structure.

One purpose of this study is to attain nearly monodisperse ANPs. This can be achieved either by sudden stop of the growth process after the nucleation reaction, or by supplying the reactant source for conservation of the saturation condition during the course of the reaction (El-Sayed et al 2005). To allow the former situation (quenching growth after nucleation), experiments were performed at $5{ }^{\circ} \mathrm{C}$ at which fast nucleation and slow

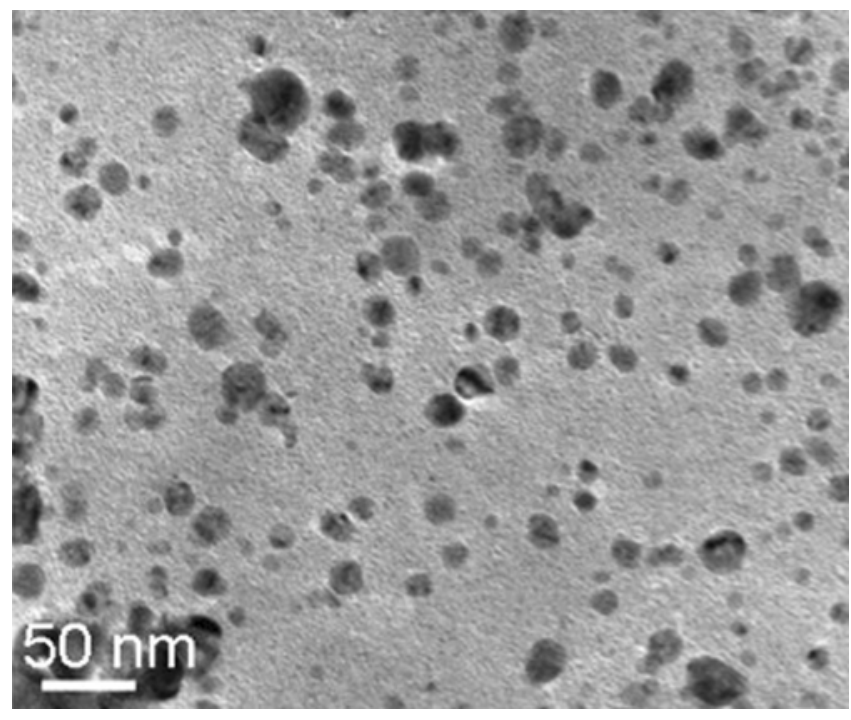

Figure 4. TEM image of mono-dispersed Ag-Ni ANPs synthesized by THPC. Some Ag-Ni ANPs contain up to 65\% Ni.

growth have occurred. TEM results showed no alloying occurrence at this temperature. Precise control of both nucleation and growth by using a strong reducing agent such as sodium borohydride was difficult. Tetrakis hydroxyl methyl phosphonium chloride (THPC) was, therefore, used as a mild reducing agent to slow down the reaction and maintain the saturation condition. Figure 4 shows monodiperse Ag-Ni ANPs obtained by THPC keeping the sample for one week at $5^{\circ} \mathrm{C}$ in a refrigerator. Although the highest Ni content of the non-equilibrium $\mathrm{Ag}_{x} \mathrm{Ni}_{1-x}$ films produced by other authors has been $56 \mathrm{wt} \%$ (Van Ingen et al 1994a, b), this study shows up to $65 \mathrm{wt} \% \mathrm{Ni}$ in some Ag-Ni ANPs produced for the first time by the method described in this research. SAD studies also confirmed the formation of amorphous structure. 


\subsection{Mechanism of Ag-Ni ANP formation}

A control experiment was performed to synthesize Ag nanoparticles (Ag NPs) using the same reduction method applied to synthesis of Ag-Ni ANPs. Figure 6(a) shows the UV-Vis absorption spectra of both Ag NP and Ag-Ni ANP. No major overall difference between the two images is observed. The UV-Vis spectrum of the Ag NP (black curve) shows characteristic surface plasmon resonance peak at $382 \mathrm{~nm}$, whereas that of Ag-Ni ANP (red curve) shows plasmon peak at $404 \mathrm{~nm}$. During synthesis, after adding the reducing agent, the intensity of the absorption peak (388 nm) increased due to formation of $\mathrm{Ag}$
NP. After $15 \mathrm{~min}$, absorption peak gradually decreased and shifted towards lower wavelengths. Then, it moved towards higher wavelengths (395 nm) after $45 \mathrm{~min}$. Shifting towards higher wavelengths could be due to oxidation. The TEM images of the Ag-Ni ANP and the approximately $5 \mathrm{~nm}$ Ag nanoparticles (Figure 6(b and c)) show the same morphology and size distribution for both systems. The Ni NP did not, however, form under the same conditions even with excess amounts of the reducing agent. Previous investigators have synthesized the Ni NP by using hydrazine (Chen and Wu 2000; Chen and Hshieh 2002; Khanna and More 2009) or sodium borohydride at temperatures between 50 and $100{ }^{\circ} \mathrm{C}$ (Hou and Kondoh 2005).
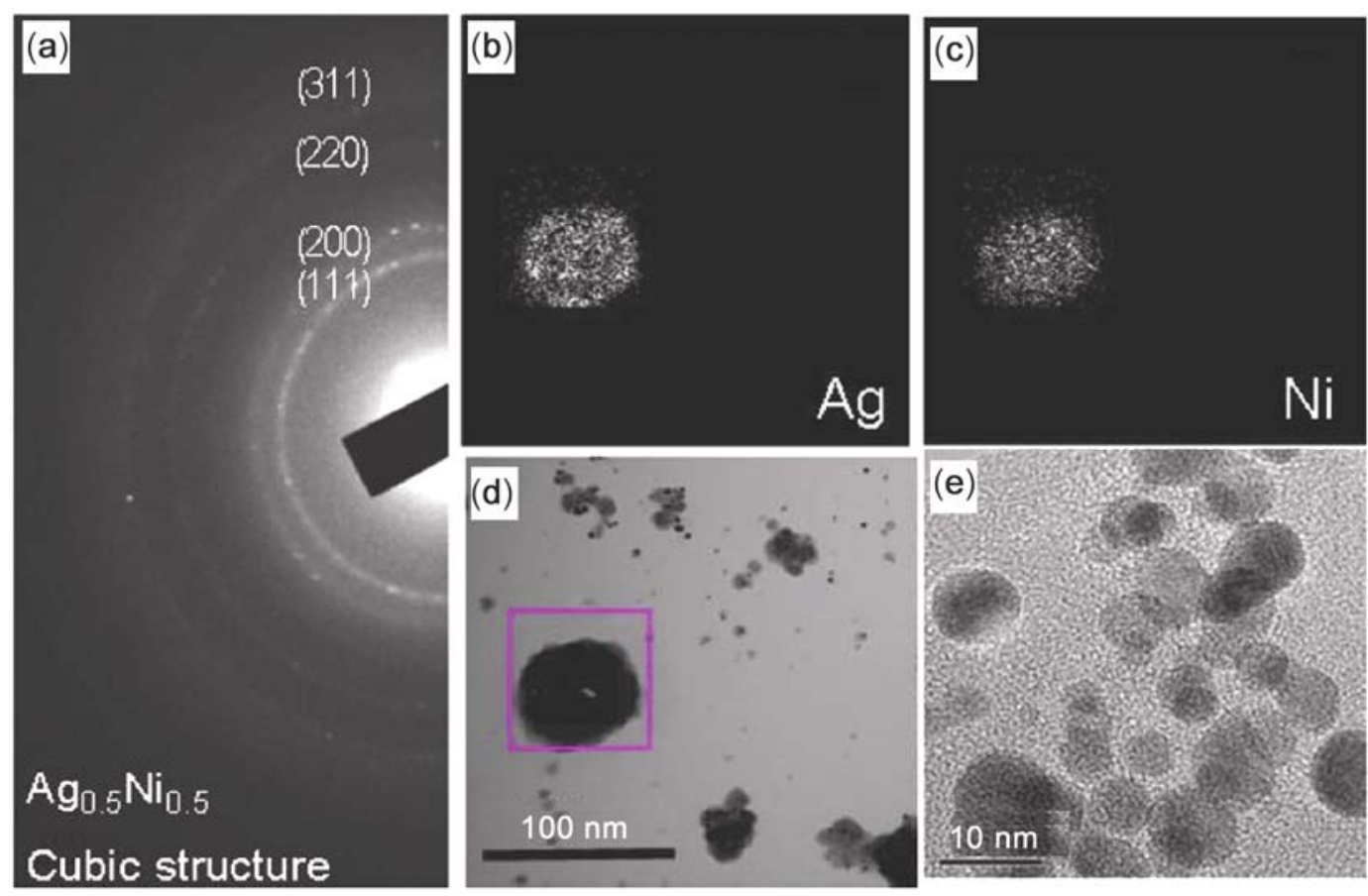

Figure 5. A typical Ag-Ni ANP particle annealed at $125^{\circ} \mathrm{C}$ for $13 \mathrm{~h}$ : (a) diffraction pattern showing cubic structure of the $\mathrm{Ag}_{0.5} \mathrm{Ni}_{0.5}$ solid solution, (b) EDS mapping of silver, (c) EDS mapping of nickel, (d) TEM micrograph of elements showing overlay and (e) TEM image indicating $\mathrm{Ag}_{0.5} \mathrm{Ni}_{0.5}$ formation.
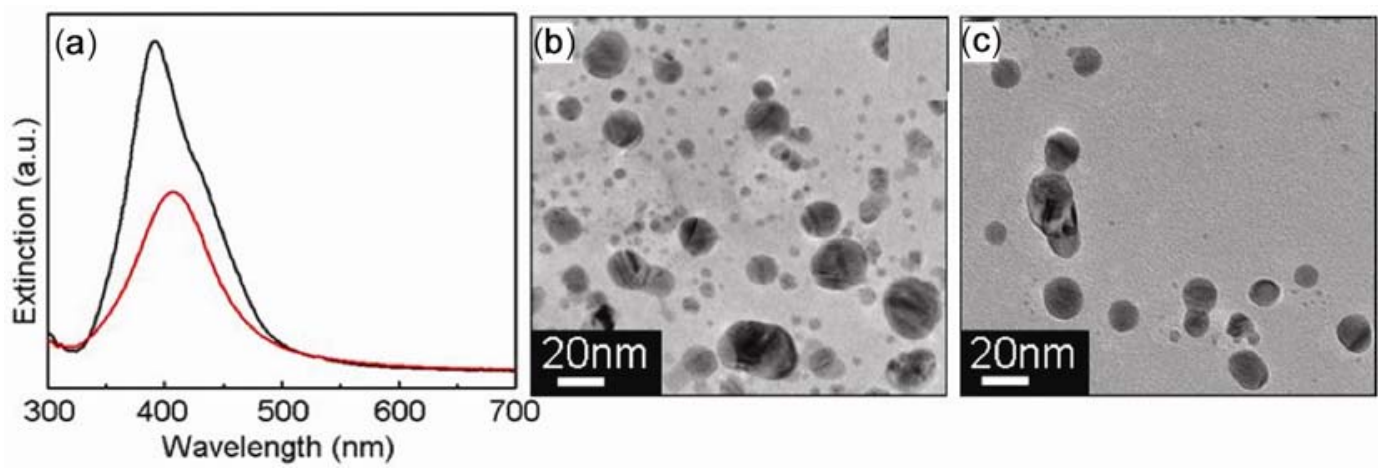

Figure 6. (a) UV-Vis spectra of Ag NP (black curve) and Ag-Ni ANP (red curve), (b) TEM image of Ag-Ni ANP and (c) TEM image of Ag NP. 


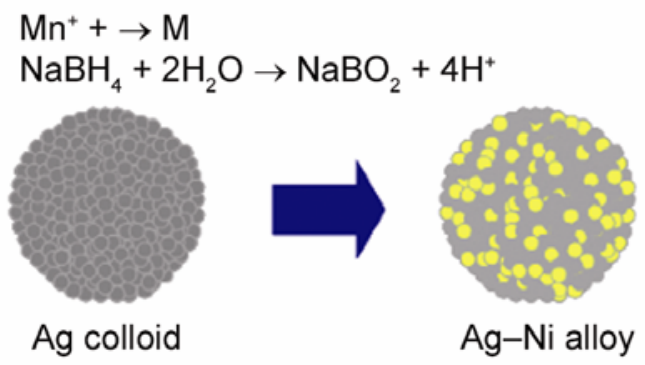

Figure 7. Schematic representation of the Ag-Ni ANP formation. M stands for a metal element.

Schematic representation of the Ag-Ni ANP formation is shown in figure 7. Standard reduction potential of Ag is higher than that of $\mathrm{Ni}$. Ag ions reduce, therefore, first to zero valent to nucleate and form Ag NP. The number of seeds produced at the nucleation stage dictates the size of the finally produced Ag-Ni ANPs. The surface and twin boundaries of the Ag NP may secondly act as catalyst (Fang and Wu 2006; Christopher and Linic 2008) acting as potential sites for reduction of the $\mathrm{Ni}$ ions. The $\mathrm{Ni}$ ions could then be reduced on the high-density planes located on the surface of the Ag NP to lower the large surface energy (Besenbacher et al 2008). Amorphous alloy may be formed when particles are so small as to have large enough surface areas. Smaller ANPs show Ni contents much higher than films reported by previous researchers (Van Ingen et al 1994a, b).

\subsection{Effect of ligands}

Ligands may change the path of formation of the alloy by affecting the surface energy of the system. Systematic adjustment of the ratio of ligands to precursors can be used to tune the size and shape of nanostructures (El-Sayed et al 2005). Controlling the size, shape and composition of ANPs, however, has been rarely studied (Samia et al 2006). Here, we investigate the effects of sodium citrate, polyvinyl alcohol (PVA) and potassium carbonate $\left(\mathrm{K}_{2} \mathrm{CO}_{3}\right)$ on size and composition of the Ag-Ni ANPs. As we mentioned in mechanism section, Ag ions reduce first. Then, absorbed ligands produce a dynamic shell that stabilizes the NPs. Ligands that bind tightly to the surface of the Ag NPs provide great steric hindrance, which slows down the rate of reduction of $\mathrm{Ni}$ ions joining the surface of the Ag NPs. This results in lower content of $\mathrm{Ni}$ in ANPs. Such a mechanism has also been reported by Peng et al (2001) for the formation of high quality CdSe nanocrystals.

Our studies revealed that addition of sodium citrate results in concomitant appearance of $\mathrm{Ag}_{2} \mathrm{O}_{2}$ together with formation of Ag-Ni ANPs. These studies showed ANPs aggregation in presence of $\mathrm{K}_{2} \mathrm{CO}_{3}$ without any silver oxidation. Composition of the ANPs also depended on the
Table 1. Influence of ligand on composition and size of the Ag-Ni ANPs.

\begin{tabular}{lcc}
\hline Ligand & Composition & ANP size \\
\hline Sodium citrate & $5-10 \% \mathrm{Ni}$ & $5-20 \mathrm{~nm}$ \\
PVA & $20-55 \% \mathrm{Ni}$ & $5-10 \mathrm{~nm}$ \\
\hline
\end{tabular}

type of ligand. With sole PVA addition, the Ag-Ni ANPs contained $20-55 \% \mathrm{Ni}$. EDS analysis showed smaller ANPs with higher nickel content. This confirmed dependency of the alloying process on the size of the ANP particles created (Yasuda and Mori 1994; Shibata and Bunker 2002). It was found out, hence, that ligand presence, precursor composition and solvent concentration affect surface energy of the particles, paths of phase transformation and the alloying yield obtained. According to the previous investigators (Wang et al 2010), $\mathrm{AgNO}_{3}$ and $\mathrm{Ni}\left(\mathrm{NO}_{3}\right)_{2}$ do not reduce in octadecylamine (ODA) solvent. Reduction of $\mathrm{AgNO}_{3}$ and $\mathrm{Ni}\left(\mathrm{NO}_{3}\right)_{2}$ to $\mathrm{Ag}-\mathrm{Ni}$ alloy was achieved, however, in presence of water in this research. Table 1 summarizes the influence of sodium citrate and PVA ligands on composition and size of the Ag-Ni ANPs.

\subsection{Stability of $\mathrm{Ag}-\mathrm{Ni}$ ANPs}

Heat stability of the Ag-Ni ANPs was tested by annealing TEM grids holding the Ag-Ni ANPs at $125^{\circ} \mathrm{C}$ for $13 \mathrm{~h}$ under vacuum. The electron diffraction pattern of a typical particle showed a cubic structure with a lattice constant of $3.83 \AA$ (figure 5a). TEM image seen in figure 5(e) indicates formation of $\mathrm{Ag}_{0.5} \mathrm{Ni}_{0.5}$ after the annealing treatment. Later, alloy structure is reported by our group for the first time. According to Vegards law, by increasing the amount of nickel in the silver structure, lattice constant will decrease. This exactly has been observed by van Ingen et al (1994a, b) for $\mathrm{Ag}_{26} \mathrm{Ni}_{74}$ (3.6916 $\AA$ ) produced by laser ablation. Although this change has rarely been reported for Ag-Ni ANPs, our observations show that its occurrence is consistent with the latter report. STEM mappings illustrated in figures 5(b and c) indicate homogeneous distribution of $\mathrm{Ag}$ and $\mathrm{Ni}$ throughout the annealed particle. TEM micrograph shown in figure 5(d) illustrates the elements and an overlay.

\section{Conclusions}

Monodisperse Ag-Ni ANPs were successfully synthesized with diameters in the range of 5-20 nm by the co-reduction of metal salts at room temperature. Gatan image filter mapping indicated that both elements existed in the produced particles. HRTEM and HAADF-STEM suggested that the ANPs produced did not have coreshell structure similar to the ones reported by previous authors. The ANPs had, instead, a relatively alloyed 
structure with some particles containing up to $65 \% \mathrm{Ni}$. Tetrakis hydroxymethyl phosphonium chloride utilization resulted in the formation of uniform ANPs.

\section{Acknowledgements}

Financial support of Prof P M Ajayan, Iran Nanotechnology Initiative Council, Research Dean of Sharif University of Technology and scientific comments of Dr Ashavani Kumar and Dr Siadati are gratefully appreciated.

\section{References}

Andrews M P and O’Brien S C1992 Phys. Chem. 968233 Banerjee R and Puthucode A 2007 Appl. Phys. Lett. 90021904

Besenbacher F, Chorkendorff I and Clausen B S 1998 Science 2791913

Chen D H and Hshieh C H 2002 J. Mater. Chem. 122412

Chen D H and Wu SH 2000 Chem. Mater. 121354

Cheng Y T and Hills R 1997 US Patent 5679471

Chinmay D, Biswas K and Sastry M 2002 Nanotechnology 13 103

Christopher P and Linic S 2008 J. Am. Chem. Soc. 13011264

El-Sayed N M 2005 Science 309404

Fang H and Wu H Y 2006 Nanotechnology 173768

Gaudry M, Cottancin E and Pellarin M 2003 Phys. Rev. B67 155409

Gheorghe P and Pantea A 1997 Metal Powder Report 5241

Ghosh S, Ray P K, Bandyopadhyay T K and Guha A K 1987 Trans. Met. Chem. 12219

Hou Y and Kondoh H 2005 Appl. Surf. Sci. 241218

Khanna P K and More P V 2009 Mater. Lett. 631384

Kiran B, Kandalam A K and Jena P 2006 J. Chem. Phys. 124 224703
Kumar A, Damle C and Sastry M 2001 Appl. Phys. Lett. 79 3314

Kusada K, Yamauchi M, Kobayashi H, Kitagawa H and Kubota Y 2010 J. Am. Chem. Soc. 13215896

Kwon K and Lee H J 1999 Appl. Phys. Lett. 75935

Lee C C, Cheng Y Y, Chang H Y and Chen D H 2009 J. Alloys Compd. 480674

Liu X, Gao F, Wang C and Ishida K 2008 J. Electron. Mater. 37210

Ma E 2005 Prog. Mater. Sci. 50413

Mondelli M, Bruné V, Borthagaray G, Ellena J, Nascimento O R, Leite C Q, Batista A A and Torre M H 2008 J. Inorg. Biochem. 102285

Morones J R, Elechiguerra J L, Camacho A, Holt K, Kouri J B, Ramírez J T and Yacaman M J 2005 Nanotechnology 16 2346

Pattabi R M, Sridhar K R and Gopakumar S 2010 J. Nanoparticles 353

Peng Z A and Peng X 2001 J. Am. Chem. Soc. 1231389

Poondi D and Singh J 2000 J. Mater. Sci. 352467

Samia A C S, Schlueter J A, Jiang J S, Bader S D, Qin C J and Lin X M 2006 Chem. Mater. 185203

Shibata T and Bunker B 2002 J. Am. Chem. Soc. 12411989

Singelton M and Nash P 1987 Bull. Alloy Phase Diagr. 8

Sondi I and Salopek-Sondi B 2004 J. Coll. Interf. Sci. 275177

Van Ingen R P, Fastenau R H J and Mittemeijer E J 1994a Phys. Rev. Lett. 723116

Van Ingen R P, Fastenau R H J and Mittemeijer E J 1994b J. Appl. Phys. 761871

Wang D, Peng Q and Li Y 2010 Nano Res. 3574

Yasuda H and Mori H 1994 Z. Phys. D31 131

Zhang Z, Nenoff T M, Leung K, Ferreira S R, Huang J Y, Berry D T, Provencio P P and Stumpf R 2010 J. Phys. Chem. C114 14309

Zhang Z, Nenoff T M, Huang J Y, Berry D T and Provenio P P 2009 J. Phys. Chem. C113 1155 